\title{
Characterization of the energy resolution and the tracking capabilities of a hybrid pixel detector with CdTe-sensor layer for a possible use in a neutrinoless double beta decay experiment
}

\author{
Mykhaylo Filipenko $^{\mathrm{a}}$, Thomas Gleixner ${ }^{\mathrm{b}}$, Gisela Anton, Jürgen Durst, Thilo Michel \\ University of Erlangen-Nuremberg, Erlangen Centre for Astroparticle Physics, Erwin-Rommel-Straße 1, 91058 Erlangen, Germany
}

Received: 27 November 2012 / Revised: 5 March 2013 / Published online: 3 April 2013

(C) The Author(s) 2013. This article is published with open access at Springerlink.com

\begin{abstract}
Many different experiments are being developed to explore the existence of the neutrinoless double beta decay $(0 v \beta \beta)$ since it would imply fundamental consequences for particle physics. In this work we present results on the evaluation of Timepix detectors with cadmium-telluride sensor material to search for $0 \nu \beta \beta$ in ${ }^{116} \mathrm{Cd}$. This work was carried out with the COBRA collaboration and the Medipix collaboration. Due to the relatively small pixel dimension of $110 \times 110 \times 1000 \mu^{3}$ the energy deposited by particles typically extends over several detector pixels leading to a track in the pixel matrix. We investigated the separation power regarding different event-types like $\alpha$-particles, atmospheric muons, single electrons and electron-positron pairs produced at a single vertex. We achieved excellent classification power for $\alpha$-particles and muons. In addition, we achieved good separation power between single electron and electron-positron pair production events. These separation abilities indicate a very good background reduction for the $0 \nu \beta \beta$ search. Further, in order to distinguish between $2 \nu \beta \beta$ and $0 \nu \beta \beta$, the energy resolution is of particular importance. We carried out simulations which demonstrate that an energy resolution of $0.43 \%$ is achievable at the $Q$-value for $0 \nu \beta \beta$ of ${ }^{116} \mathrm{Cd}$ at $2.814 \mathrm{MeV}$. We measured an energy resolution of $1.6 \%$ at a nominal energy of $1589 \mathrm{keV}$ for electron-positron tracks which is about two times worse that predicted by our simulations. This deviation is probably due to the problem of detector calibration at energies above 122 $\mathrm{keV}$ which is discussed in this paper as well.
\end{abstract}

\footnotetext{
a e-mail: mykhaylo.filipenko@physik.uni-erlangen.de

b e-mail: thomas.gleixner@physik.uni-erlangen.de
}

\section{Introduction}

1.1 Neutrinoless double beta decay

The neutrinoless double beta decay is a hypothetical weak decay process in which two neutrons of a nucleus decay to two protons and two electrons:

$2 n \rightarrow 2 p+2 e^{-}$

This decay is forbidden in the standard model of particle physics since the lepton number is not conserved. Additionally, this process can occur only if neutrinos are massive Majorana-particles and consequently, the neutrino has to be its own antiparticle [1].

The half-life for nuclides which can undergo this process, like ${ }^{76} \mathrm{Ge},{ }^{136} \mathrm{Xe}$ or ${ }^{48} \mathrm{Ca}$ for example, can be calculated by the formula [2]

$T_{0 v}^{-1}=G_{0 v}\left(Q_{\beta \beta}, Z\right)\left|M_{0 v}\right|^{2}\left\langle m_{v}\right\rangle^{2}$

where $G_{0 v}$ is the phase space volume, which depends on the $Q$-value $\left(Q_{\beta \beta}\right)$ of the decay and the charge number $Z$ of the nuclide. $M_{0 v}$ is the $0 v \beta \beta$ transition matrix element and $\left\langle m_{v}\right\rangle$ the effective Majorana-neutrino mass. If, according to claims of a past experiment [3], an effective neutrino mass of $\left\langle m_{v}\right\rangle<0.39 \mathrm{eV}$ is assumed, half-lifes of $T_{\nu 0}>10^{25} \mathrm{a}$ are expected. Therefore, an experimental observation of this process is highly challenging and could not be achieved until now; but since an observation of $0 \nu \beta \beta$ would provide insight into new fundamental physics beyond the standard model [1], many experiments are running or being developed to achieve an experimental proof for $0 \nu \beta \beta$.

In an experiment the $0 \nu \beta \beta$ is identified by measuring the sum energy of the two electrons, which is equal to the full $Q$-value of the decay [4]. The main goal is to construct a detector with sufficient resolution and high mass. High mass is indispensable to achieve a good sensitivity on the effective 


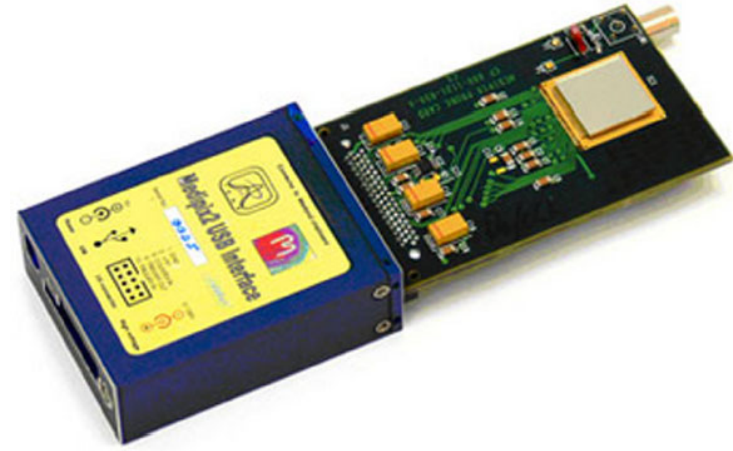

Fig. 1 The Timepix detector with a USB-Readout [9]

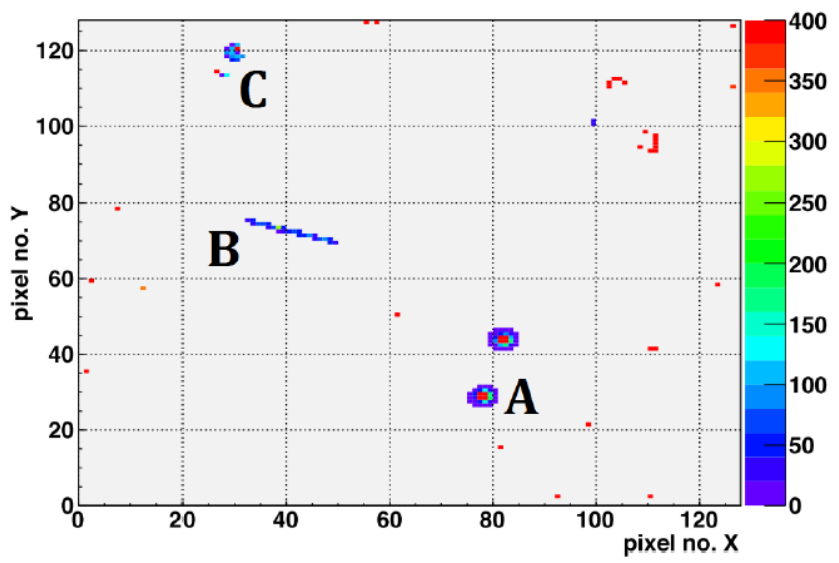

Fig. 2 Image of deposited energy in the pixels of the CdTe Timepix detector during an exposure time of $5 \mathrm{~s}$. The color denotes the energy deposition in $\mathrm{keV}$. One can deduce that two $\alpha$-particles $(A)$, a muon $(B)$ and an electron $(C)$ have interacted with the sensor

neutrino mass which is the lowest effective neutrino mass $\left\langle m_{v}\right\rangle$ that can be set as the limit with an experiment of mass $M$ at an exposure time $T$ if no signal event was measured. The sensitivity can be approximated by the formula [5]:

$\left\langle m_{v}\right\rangle=K\left(\frac{b \Delta E}{M T}\right)^{\frac{1}{4}}$

An excellent energy resolution $\Delta E$ is not only required to enhance the sensitivity but also to discriminate $0 \nu \beta \beta$ against the neutrino accompanied double beta decay [4]. In order to avoid energy loss at insensitive detector boundaries, most experiments use a detector which contains the considered nuclide in the sensor material. $K$ is an isotope depending constant. As the mass $M$ and the exposure time $T$ are limited by practical or cost issues and the energy resolution by the fano-limit, the only way to increase sensitivity further is to reduce the background rate $\mathrm{b}$ as good as possible. The background in the region of interest is mainly provided by $\alpha$-particles, muons and electrons.

In this paper we investigated the concept of active background reduction by the tracking capabilities of Timepix de- tectors with CdTe sensors: The Timepix (Fig. 1) is a hybrid semiconductor pixelated imaging detector which is explained below. When a charged particle propagates through the sensor layer it induces electron-hole pairs proportional to the amount of energy which was deposited by the particle. By applying a bias voltage over the sensor layer, the electrons drift towards the electrodes where the induced charge is collected. If a particle is stopped within the sensor layer completely, its total kinetic energy is deposited. Therefore, if $0 v \beta \beta$ takes place within the sensor layer, the total energy of the electrons can be measured. Indeed, there are several possible isotopes for this purpose in $\mathrm{CdTe}$ [7] but as ${ }^{116} \mathrm{Cd}$ has the highest $Q$-value of $2.814 \mathrm{MeV}$ [8], which is larger than the highest $\gamma$-energy $(2.614 \mathrm{MeV})$ from a natural decay chain, enriched cadmium should be used as the decaying isotope. The advantage of the pixelation (in contrast to CdZnTe Coplanar Grid detector) is that a topological pattern is produced for each event (Fig. 2). This information might be used for event identification and active background rejection. As preliminary measurements show, $\alpha$-particles produce a round shaped pattern (Fig. 2 A) and muons produce straight lines (Fig. 2 B). Hence, they are very unlikely to be confused with the pattern of $0 \nu \beta \beta$, which we estimated by simulations. A detailed discussion on the identification of this sort of background will be presented elsewhere. The most severe problem, which we want to focus on in this paper, is to distinguish between single electrons and $0 \nu \beta \beta$ events (Fig. 3(a) and (b)). We used simulation data to study the particular properties of both event types. Afterwards we trained artificial neural networks (ANNs) and estimated their performance first on simulated and then on experimental data.

\subsection{The timepix detector}

Our Timepix detector comprises a $1 \mathrm{~mm}$ thick CdTe sensor with ohmic contacts bump-bonded to an ASIC of 65536 pixels. The Timepix ASIC has been developed by the Medipix collaboration [6] in cooperation with the EUDET project in IBM $0.25 \mu \mathrm{m}$ CMOS technology. Each pixel has a size of $55 \mu \mathrm{m}$. The pixels are organized in a matrix of 256 rows and 256 columns thus giving an active area of $1.4 \times 1.4 \mathrm{~cm}^{2}$. In our detector only every second pixel is bump-bonded to the sensor layer wherefore the effective pixel size is $110 \mu \mathrm{m}$. The CdTe sensor is fully depleted with an electric potential difference of $500 \mathrm{~V}$ between the common electrode (facing the source) and the pixel electrodes. Electron-hole pairs are produced by ionizing particles in the sensitive pixel volume and drifted towards the pixel electrodes where they influence currents due to their drift motion. Each sensor pixel is connected by an indium-tin bond to the input electrode of an electronics cell in the ASIC. In each pixel cell the influenced current signal is converted to a triangular shaped voltage 


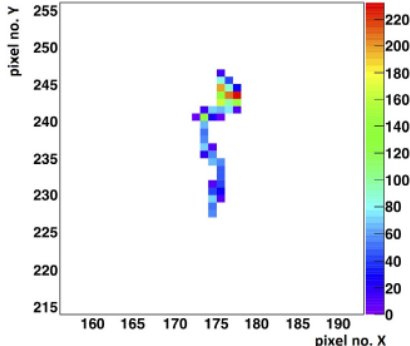

(a) an electron event (b) a $0 v \beta \beta$ event

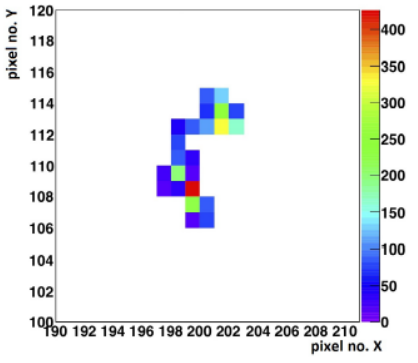

Fig. 3 Examples for pattern of a simulated electron (a) and a simulated $0 \nu \beta \beta$ (b) event in ${ }^{116} \mathrm{Cd}$. The color denotes the energy deposition in $\mathrm{keV}$

pulse by a charge sensitive amplifier of Krummenacher type. The shaping time is approximately $120 \mathrm{~ns}$. The length of the falling edge of the amplifier is in the order of microseconds, depending on the energy deposited in the pixel. This voltage pulse is compared to a globally adjustable threshold in a leading-edge discriminator in each pixel. The minimum threshold that can be set with negligible amount of noise-triggered pixels is approximately $5.5 \mathrm{keV}$. The gain of the preamplifier, the falling edge of the preamplifier and the threshold can be set externally by a DAC. The DAC-values are referred as PreAmp, Ikrum and THL, respectively. After the amplifier the processing is purely digital and depends on the operation mode which is chosen. Each pixel can be configured in one of three modes of digital operation. We used only the spectroscopic mode which is called "timeover-threshold" (TOT). It is described in the calibration section.

\section{Simulations}

The simulations were performed with the in-house developed Monte-Carlo simulation ROSI, which is based on EGS4 and has a low energy extension with the interaction codes LSCAT [10]. Each event is simulated independently by propagating the corresponding particles (photons, electrons, holes) through the sensor and calculating the detector response (i.e. the energy measured in each pixel). The initial momentum of the particles is calculated with decay 0 [11].

We used the simulations to estimate the detector response to $0 \nu \beta \beta$ events. A typical pattern for such an event is shown in Fig. 3(b). The two main aspects of the detector performance concerning $0 v \beta \beta$ experiments are the energy resolution, which is required to distinguish between the regular double beta decay $(2 v \beta \beta)$ and $0 \nu \beta \beta$, and the spatial resolution which determines the tracking quality. Both properties depend on detector parameters like the pixel size, the thickness of the sensor layer or the bias voltage. The energy resolution can improve with increasing pixelsize and increasing

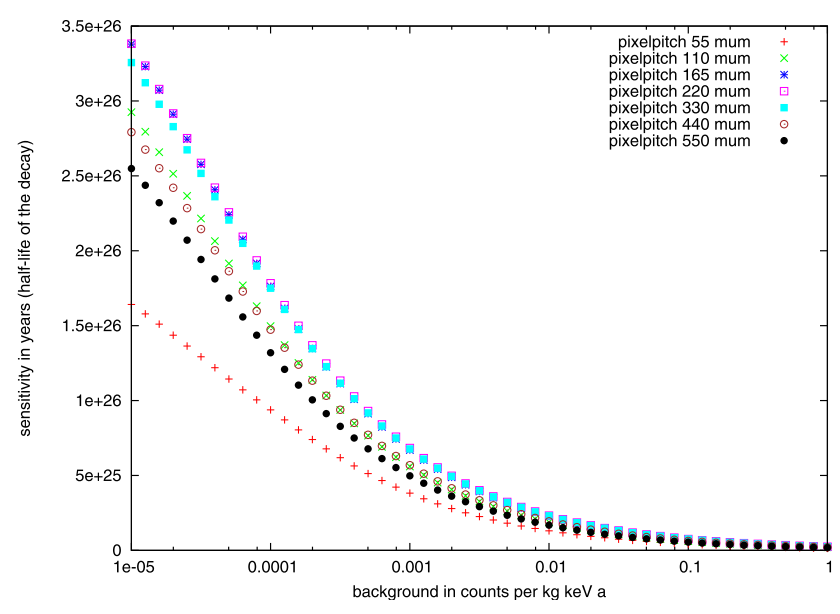

Fig. 4 Sensitivity versus background rate of a CdTe-Timepix detector with a $3 \mathrm{~mm}$ thick CdTe sensor. The best sensitivity can be achieved with a pixelsize of $165 \mu \mathrm{m}$

bias voltage since effects like charge sharing (leakage of the charge to neighbor pixel by repulsion and diffusion) [12] have smaller contribution. In contrast, a large pixel size provides less tracking information and therefore a compromise is needed. In Fig. 4 the simulated sensitivity at given background rate for a $3 \mathrm{~mm}$ thick sensor is shown which suggests that the best sensitivity can be achieve with pixels of either $165 \mu \mathrm{m}$ or $220 \mu \mathrm{m}$ size. However, a smaller pixel size is apparently preferable for tracking. As this paper focuses on the performance of the Timepix performance with experimental data, the details of this evaluation will be discussed in a future paper. Although it turned out that a reasonable tradeoff between the two effects is achieved at a pixelsize of $165 \mu \mathrm{m}$, we used a detector with $110 \mu \mathrm{m}$ pixel size detector for the experiments because only those were available to us.

A simulated sum energy spectrum-i.e. the energy deposited by the two electrons from the ${ }^{116} \mathrm{Cd}$ decay-around the $Q$-value is shown in Fig. 5 for a Timepix with $165 \mu \mathrm{m}$ pixelsize and a thickness of $3 \mathrm{~mm}$. The simulation yields a resulting energy resolution of $0.43 \%\left(\frac{\sigma}{E}\right)$ at the $Q$-value of $2.814 \mathrm{MeV}$ which allows a reasonable separation of $0 \nu \beta \beta$ events from the $2 \nu \beta \beta$ spectrum. The $0 \nu \beta \beta$ would be observed at lower energies compared to the full $Q$-value due to various mechanisms of charge loss within the detector. For the simulations we assumed a half-life of $2.75 \times 10^{25}$ years, a detector mass of $400 \mathrm{~kg}$ with $90 \%$ enriched ${ }^{116} \mathrm{Cd}$ observed over 3 years and well-calibrated detectors. In fact, the calibration of the Timepix is non-trivial and will be discussed in Sect. 3. Especially, a calibration up to energy depositions of several hundred $\mathrm{keV}$ per pixel is required since such depositions often have non-negligible contributions to the total energy of the event: In Fig. 6 the contribution of the deposited energy per pixel $P\left(E_{d e p}\right)$ to the total detected event energy is plotted against the energy deposition per pixel $E_{d e p} . P\left(E_{d e p}\right)$ is defined as 


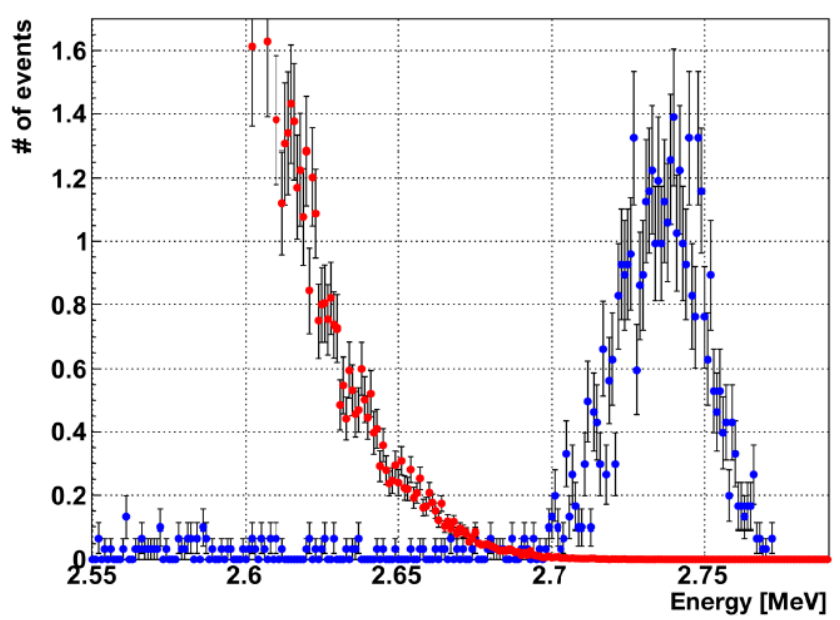

Fig. 5 The simulated spectrum of $2 \nu \beta \beta$ (red) and $0 \nu \beta \beta$ (blue) events around the $Q$-value. The bin size is $1 \mathrm{keV}$. The assumptions of the simulation are given in the text

$P\left(E_{\text {dep }}\right)=\frac{1}{N_{\text {events }}} \cdot\left(\frac{E_{\text {dep }}}{Q} \cdot N_{\text {pix }}\left(E_{\text {dep }}\right)\right)$,

where $N_{\text {events }}$ is the number of simulated events and $N_{\text {pix }}\left(E_{\text {dep }}\right)$ is the number of pixels (summed over all events) which had an energy deposition of $E_{d e p}$. The bin size in the histogram is $4 \mathrm{keV}$. We see that even depositions up to $600 \mathrm{keV}$ are relevant.

As stated in the introduction single electrons produce pattern which may be very similar to the pattern of $0 v \beta \beta$ and therefore a unique event identification can hardly be performed. Nevertheless this problem can be addressed by several pattern identification techniques. We used Artificial Neural Networks (ANNs) which were implemented with the open source library FANN [13]. The networks had a feed forward structure with the following parameters:

- 10 input units

- 1 output unit

- 5 hidden layers

- between 20 and 200 neurons per layer

$-\tanh (0.3 x)$ as activation function

- Resilient Propagation as learning algorithm.

We used the following properties of the track pattern as features for the ANN input:

1. The number of pixel with $E>E_{t h}$, where $E_{t h}$ is the energy threshold for each pixel.

2. The distance between the energy weighted and the energy unweighted centroids of the coordinates of the triggered pixels.

3. The energy of a two pixel structure that can mark the begin of a single electron track.

4. The energy of a three pixel structure that can mark the begin of a single electron track.

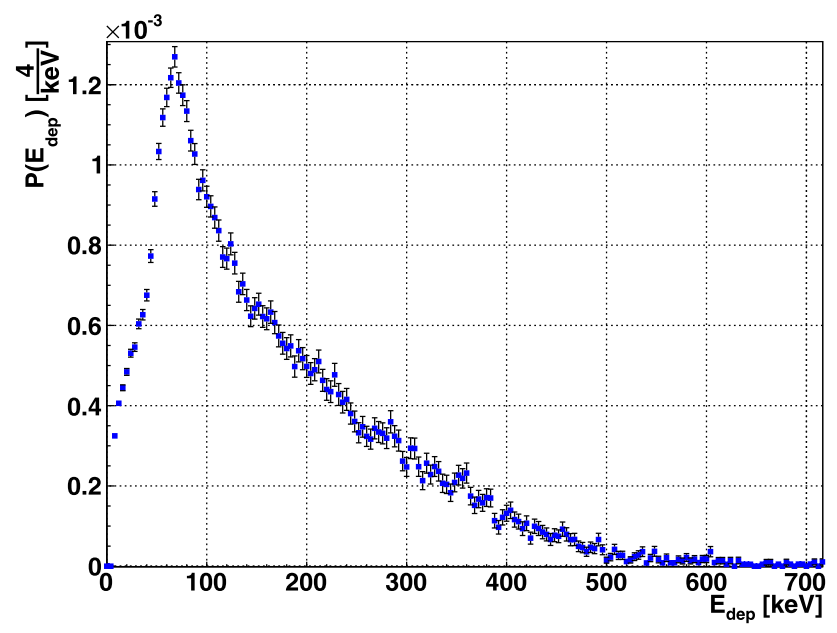

Fig. 6 The contribution of a particular energy deposition per pixel to the full energy of an event. The error bars are Poissonian

5. The length of a structure that can mark the begin of a single electron track.

6. The maximum distance of pixels above $250 \mathrm{keV}$.

7. The number of pixels with an energy sum below 185 $\mathrm{keV}$ with their neighbor pixels.

8. The number of straight lines above a certain length in a track.

9. The number of pixels with only two triggered pixel touching the pixel from the side, which form a straight line together.

10. The number of pixels with only two triggered pixel touching the pixel from the side, which form a triangle together.

The networks are trained on the same number of simulated single electron and $0 \nu \beta \beta$ events. Each event is assigned a ranking $R$ between 0 and 100 (which is not a physical quantity) and a particular cut value $c(c \in[0 ; 100])$ is chosen. If the rating of an event $R$ is smaller than the cut parameter $c$, it is classified as a single electron event and vice versa. The identification performance of the networks on independent simulated data sets ${ }^{1}$ is shown on Fig. 7. On the $\mathrm{x}$-axis the value of the cut parameter is plotted and on the $\mathrm{y}$-axis the part of events which have a rating $R<c$. That means, if we fix a particular value for $c$, for instance 70 , we can reject about $90 \%$ of the single electron background but will lose $40 \%$ of the $0 \nu \beta \beta$ events (because of classification errors). In order to test the performance of this method on real data, we carried out the experiment which is presented in Sect. 4.

\footnotetext{
${ }^{1}$ Independent in this context means, that the data which was used for the performance analysis was not used for the network training beforehand.
} 


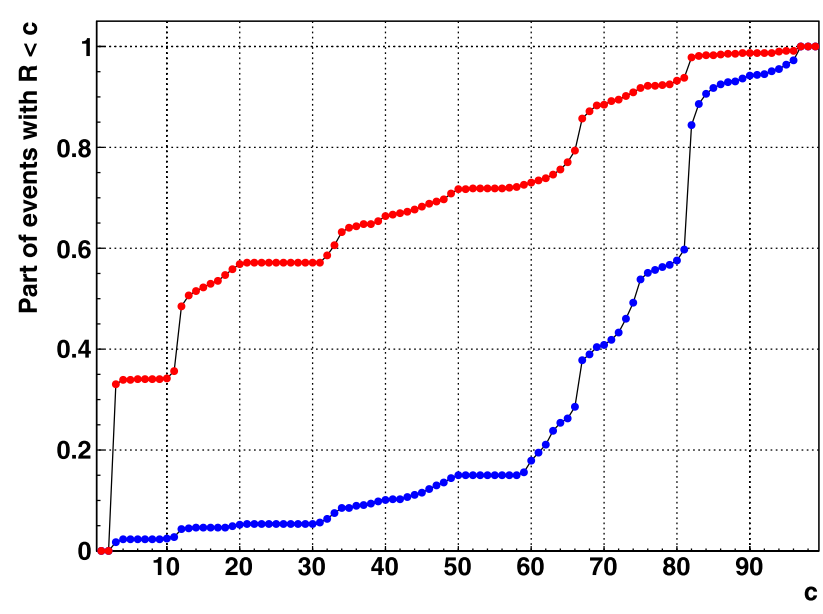

Fig. 7 Performance of artificial neural networks on simulated data from single electron (red) and $0 \nu \beta \beta$ (blue) events

\section{Calibration and energy resolution}

The Timepix detector enables the measurement of the energy deposited in each pixel by running in the so called "time-over-threshold" (TOT) mode. Energy deposition in a pixel leads to the generation of electron-hole pairs. An electric field, which is applied over the sensor layer, drifts these charges towards the pixel electrodes. While the charges are drifted, they induce a signal in the pixel electrodes that is integrated and converted to a voltage signal in the preamplifier. A particular threshold level (THL) is chosen. When the signal rises above this threshold, the counting of clock pulses is started and continued until the signal falls below the threshold again. The number of counts-called time over threshold (TOT) - depends non-linearly on the energy and an interdependency between both has to be determined. To perform this task we used the method described in [14]: The peaks in the TOT spectra ${ }^{2}$ of known photon radiation sources are fitted with Gaussian distributions and the mean of the distribution is taken for a TOT versus energy calibration. From measurements with different $\mathrm{X}$-ray sources the diagram in Fig. 8 was obtained. The real energy depositions by the $\mathrm{X}$ ray photons have to be estimated by a simulation since they are slightly shifted to lower energies (compared to the energies of the absorbed photons) due to trapping, charge sharing and other effects of charge loss [12]. The TOT(E) interdependency is approximated by [14]:

$\operatorname{TOT}(E)=a \cdot E+b+\frac{c}{E-t}$

The calibration can be performed with one parametrization for all pixels (global calibration) or for every pixel in-

\footnotetext{
${ }^{2}$ The spectra that we used were single clustering spectra. That means, that only the data of pixels is used which had no triggered neighbor pixels during the same frame.
}

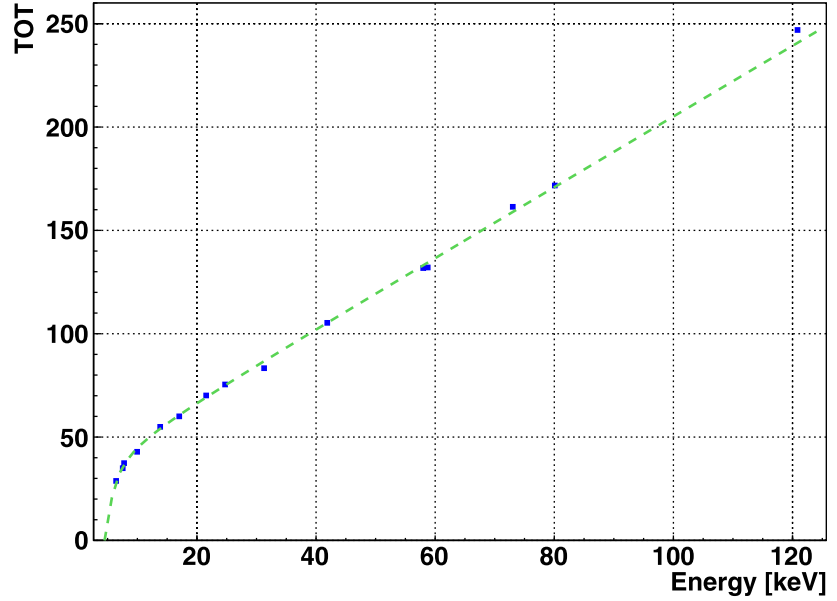

Fig. 8 Interdependency between TOT and energy; data points are from illumination with monoenergetic X-rays sources. The dotted curve is a fit assuming $\operatorname{TOT}(E)=a \cdot E+b+\frac{c}{E-t}$ with parameters $a, b, c$ and $t$ common for all pixels (global calibration)

dividually (pixel-by-pixel calibration). For the global calibration we used all points as shown in Fig. 8; for the pixelby-pixel calibration we used the L- and K-line complexes ${ }^{3}$ of gadolinium $(6.43 \mathrm{keV}, 41.88 \mathrm{keV})$, the K-line complex of molybdenum (17.03 keV), the $59.54 \mathrm{keV}$-line of ${ }^{241} \mathrm{Am}$ and the $122.06 \mathrm{keV}$-line of ${ }^{57} \mathrm{Co}$. We calibrated and used a Timepix with a $1 \mathrm{~mm}$ thick CdTe sensor layer, ohmic contacts and a pixel size of $110 \mu \mathrm{m}(128 \times 128$ pixel $)$ which was delivered by X-ray Imaging Europe $\mathrm{GmbH} \mathrm{XIE}^{4}$ [15]. The bias voltage was $500 \mathrm{~V}$, the clock frequency $80 \mathrm{MHz}$ and the most important DAC settings: Ikrum 10 (return to zero time of the preamplifier), THL 190 (threshold level), which corresponds to an energy threshold of about $5.4 \mathrm{keV}$, and PreAmp 210 (preamplifier gain). For the data acquisition a USB-Readout 2.0 [9] and the Pixelman software package v2.0.3 were used [16].

After reconstructing the energy spectra (see Fig. 9 for example) for single pixel hits ${ }^{5}$ we investigated the energy resolution ( $\frac{\sigma}{E}$ of the fitted Gaussians) of the detector for various peaks. The results are shown in Fig. 10. The blue points are the energy resolution if a global calibration is used for energy reconstruction, the red points if a pixel-by-pixel calibration is used and the brown points are the results of simulations which take into account only the physics within the sensor layer but no other effects of broadening in the readout electronics. Hence, the brown points correspond to the

\footnotetext{
${ }^{3}$ The L- and $\mathrm{K}$-line complexes are an overlay of the $\alpha$ - and $\beta$-lines since these cannot be resolved individually.

${ }^{4}$ www.xi-europe.de, Freiburger Materialforschungszentrum, StefanMeier-Straße 21, D-79104 Freiburg i. Br.

${ }^{5}$ Single pixel hits means that the energy of the incident photon is deposited in one pixel in such a way that no neighboring pixel is triggered.
} 


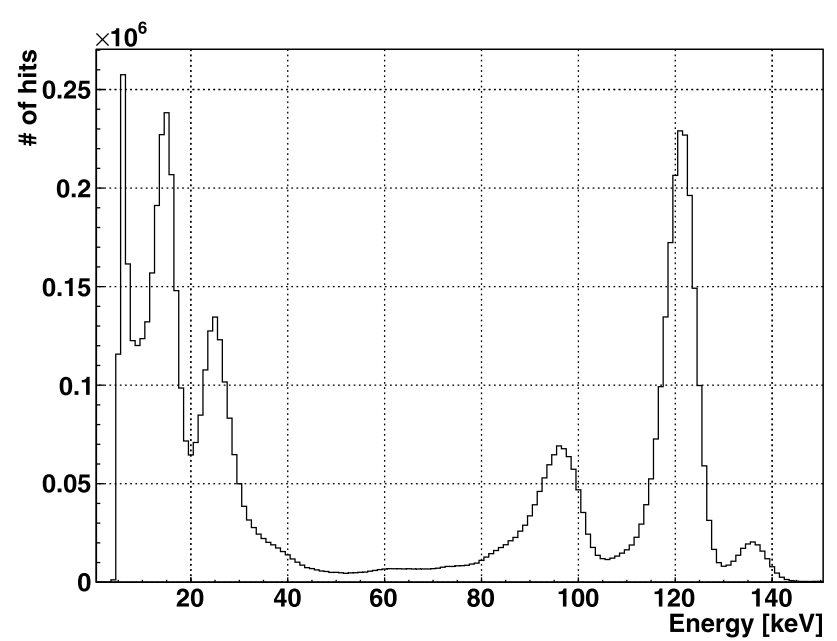

Fig. 9 The energy deposition spectrum of photons from a ${ }^{57} \mathrm{Co}$ source, reconstructed with a pixel-by-pixel calibration

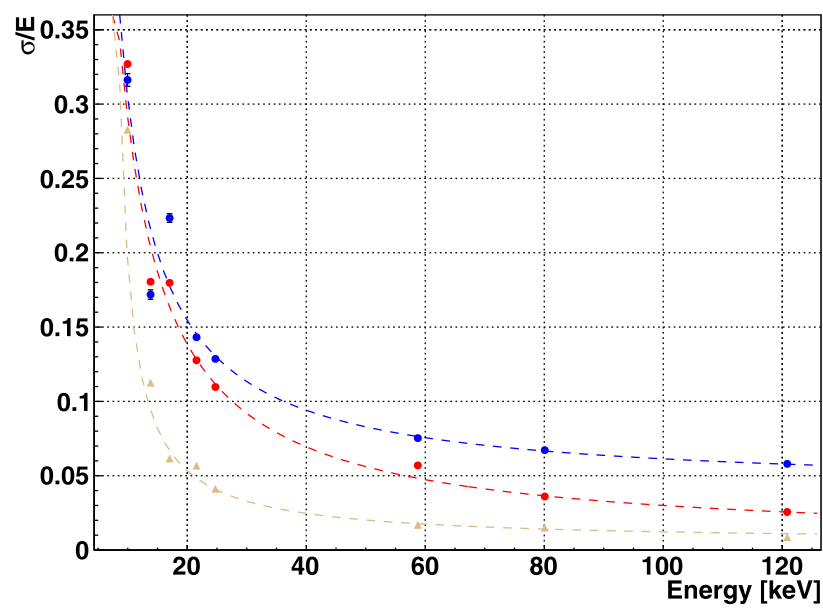

Fig. 10 The energy resolution for K- and L-Shell X-rays and radioactive $\gamma$-lines with a global calibration (blue); with a pixel-by-pixel calibration (red) and for ROSI simulation data (brown) with IKrum 10 and THL 190. For further explanations see text

physical possible limits set by the detection material which theoretically could be achieved. We fitted a function of the form

$\frac{\sigma}{E}(E)=\alpha+\frac{\beta}{E-\gamma}$

to the data in Fig. 10 to estimate the energy resolution for single pixel hits at high energy depositions per pixel. The energy resolution limit $\alpha$ was determined to be $\alpha=4.10 \%$ for the global calibration, $\alpha=1.01 \%$ for the pixel-by-pixel calibration and $\alpha=0.54 \%$ for the ideal detector. We compared the performance of the detector to the simulations which included the noise of readout electronics. The deviations be-

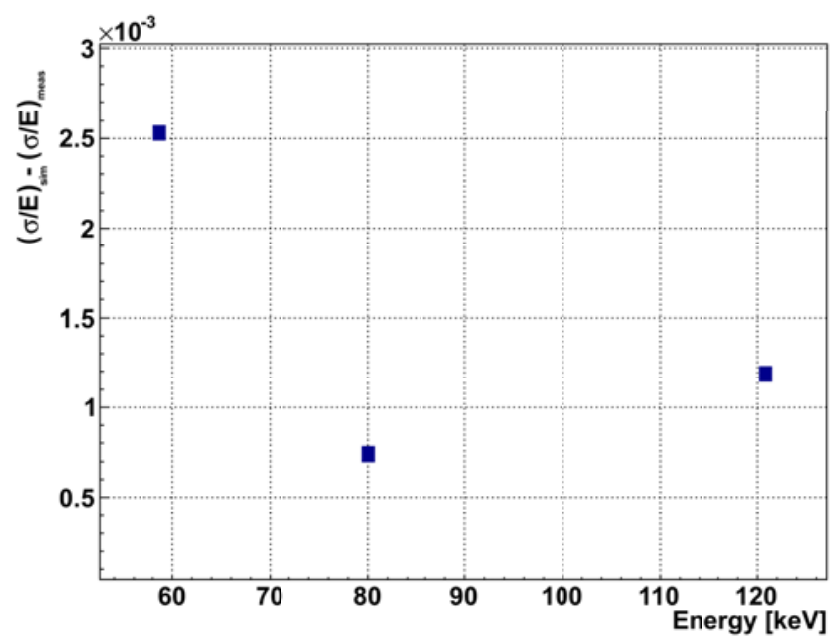

Fig. 11 Difference of measured relative energy resolution and simulated relative energy resolution versus photon energy. Statistical errors are small and not visible

tween simulation and theory are shown in Fig. $11 .^{6}$ We obtain a good agreement between experiment and simulation.

Since the shape of the voltage signal used in the integrator to calculate the TOT depends on the working parameters of the detector (Ikrum, THL and the bias voltage), we investigated the influence of different parameter sets for Ikrum and THL on the energy resolution. We could achieve an improvement in the energy resolution as presented in Table 1 by going from the usual settings that we used (Ikrum 10, THL 190) to the lowest possible Ikrum (04) and a THL of 210. The dependence on the bias voltage is presented in Fig. 12. The blue points are the energy resolution for the $59.54 \mathrm{keV}$ peak of ${ }^{241} \mathrm{Am}$ and the red points for the 122.06 $\mathrm{keV}$ peak of ${ }^{57} \mathrm{Co}$. As expected, the energy resolution improves with higher bias voltage because the effect of charge sharing [12] is reduced with higher bias voltage. The highest voltage that we could use without exceeding a critical value of the leakage current $(40 \mu \mathrm{A})$ was about $800 \mathrm{~V}$. Nevertheless going up with the bias voltage leads to an additional negative effect: The number of useless pixels (pixels which are permanently counting) increases rapidly (Fig. 13). The dependence of the number of useless pixels on the bias voltage can roughly be approximated by a power-law (red curve). We didn't investigate this problem in more detail but a reasonable explanation could be the following: It is wellknown that the leakage current rises with the voltage. However, the leakage current in every pixel is slightly different due to sensor and ASIC fabrication. Some pixels have always a higher leakage current because of inhomogeneities in the CdTe-crystal and the leakage-current compensation.

${ }^{6}$ For this plot as for some other in this paper the error bars are small and invisible because for every point the statistics is very high which is the number of pixels on the matrix (16384). 


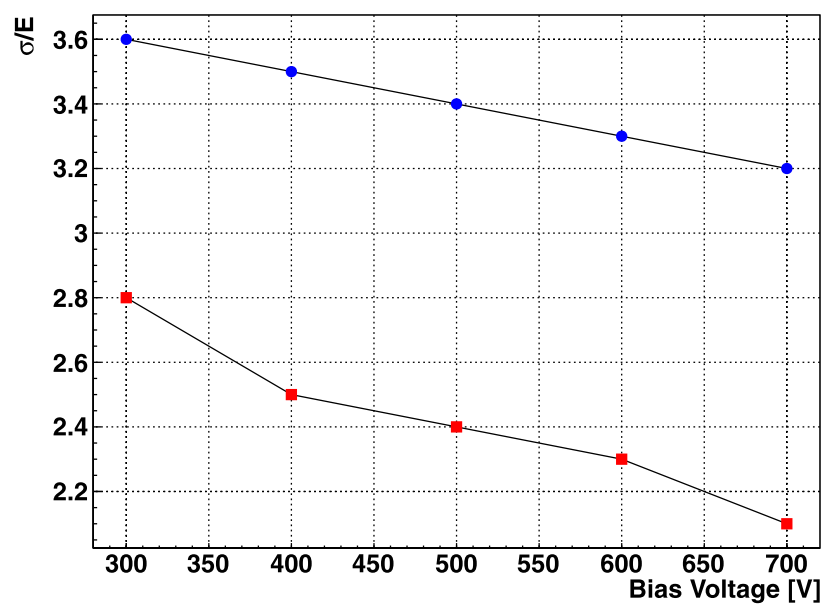

Fig. 12 Energy resolution versus bias voltage for ${ }^{241} \mathrm{Am}$ (at 59.54 $\mathrm{keV})$ (blue) and ${ }^{57} \mathrm{Co}$ (at $122.06 \mathrm{keV}$ ) (red) with a pixel-by-pixel calibration for IKrum 04 and THL 210. Statistical errors are very small and thus not visible

Table 1 The measured relative energy resolution for ${ }^{241} \mathrm{Am},{ }^{133} \mathrm{Ba}$ and ${ }^{57} \mathrm{Co}(122.06 \mathrm{keV})$ given in \% for two different DAC settings; the bias voltage was $500 \mathrm{~V}$

\begin{tabular}{llll}
\hline DACs & ${ }^{241} \mathrm{Am}$ & ${ }^{133} \mathrm{Ba}$ & ${ }^{57} \mathrm{Co}$ \\
\hline IKrum 10, THL 190 & 5.6 & 3.5 & 2.5 \\
IKrum 04, THL 210 & 3.4 & 3.2 & 2.3 \\
\hline
\end{tabular}

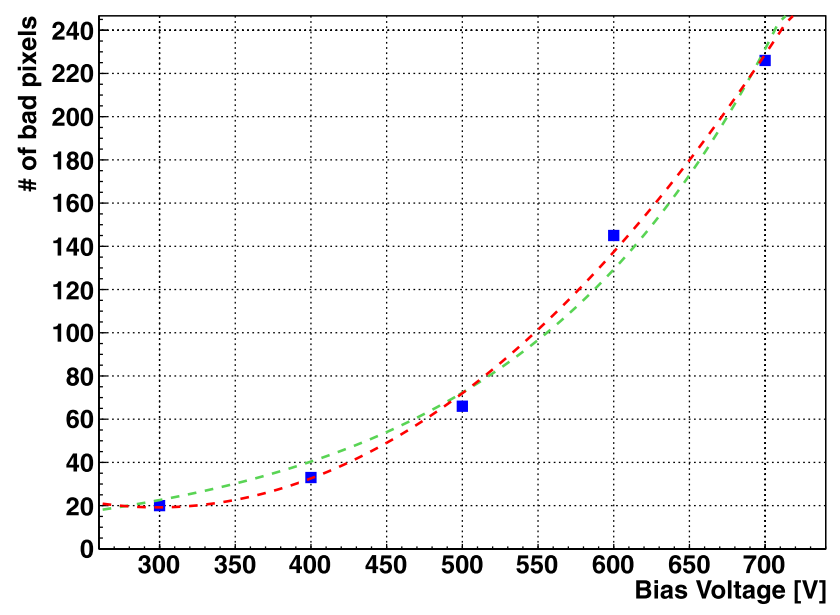

Fig. 13 Number of useless pixels on the detector matrix as function of the bias voltage. A power law (red) of the form $N(V)=a \cdot(V-b)^{2}+c$ and an exponential function (green) of the form $N(V)=a \cdot \exp (-b \cdot V)$ are shown as possible fit functions. The detector contains 16384 pixels in total

Once, the noise produced by the leakage current rises above the threshold level in these particular pixels, the pixels are permanently counting.

The highest photon energy that could be used for a pixelby-pixel calibration on a practical timescale was $122.06 \mathrm{keV}$.

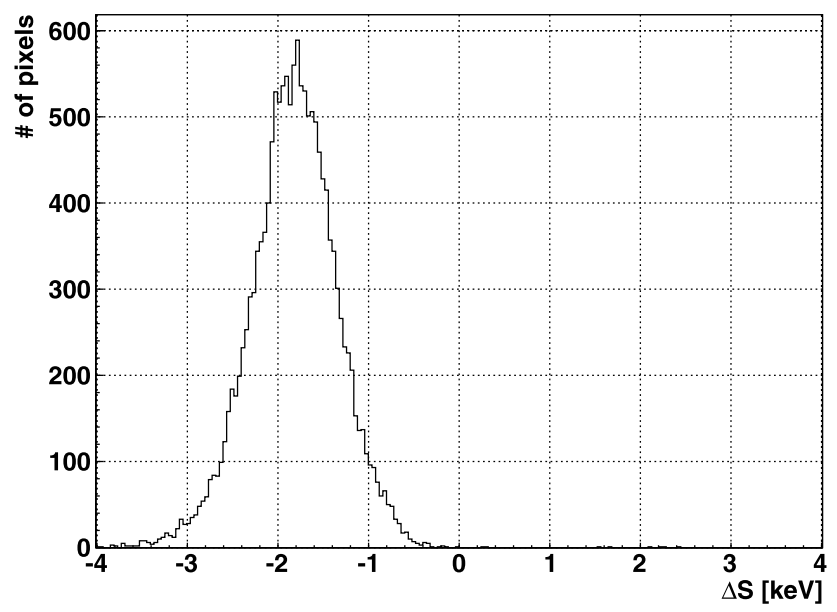

Fig. 14 The distribution of $\Delta S$ at the ${ }^{241}$ Am peak (59.54 keV) for all pixels. It is (almost) a Gaussian distribution around $-1.88 \mathrm{keV}$ with a width of $\sigma=0.43 \mathrm{keV}$

For higher energies the efficiency for single pixel hits is far too small. This has two reasons: Firstly, the absorption efficiency for a thick $1 \mathrm{~mm}$ CdTe sensor is smaller than $10 \%$. Secondly, with higher photon energies the travel distance of the photoelectron within the CdTe increases and is usually larger than the pixelsize of $110 \mu \mathrm{m}$. This means, that single pixel hits, which are needed for calibration, become more and more unlikely with increasing energy. We measured one week to achieve 300 counts over the whole matrix at the $356.02 \mathrm{keV}$ peak of ${ }^{133} \mathrm{Ba}$. Assuming that we can take data 50 times faster with a new read-out and that at least 200400 counts are required in one pixel for a reliable calibration, a total measurement time of about $\frac{16384}{50.52} \approx 6.3$ years is needed. Therefore, a calibration cannot be done this way on practical timescales.

However, as electrons can often deposit energies in the $\mathrm{MeV}$ range in one pixel, it is important to estimate the quality of the calibration beyond the highest pixel-by-pixel calibration point. Beyond an energy of $122.06 \mathrm{keV}$ we used four peaks with energies at $136.47 \mathrm{keV}\left({ }^{57} \mathrm{Co}\right), 184.35 \mathrm{keV}$ ( ${ }^{137} \mathrm{Cs}$ Compton backscattering), $238.63 \mathrm{keV}$ and 356.02 $\mathrm{keV}\left({ }^{133} \mathrm{Ba}\right)$. For this purpose we define the quantity $\Delta S(E)$ which is the difference between the expected peak position and the reconstructed peak position with a pixel-by-pixel calibration at a peak energy E:

$$
\begin{aligned}
\Delta S(E)= & \text { Expected peak position at energy } E \\
& - \text { Reconstructed peak position at energy } E
\end{aligned}
$$

The distribution of $\Delta S(59.54 \mathrm{keV})$ among all pixels of the matrix is shown in Fig. 14. For other energies the distribution has a similar shape. For an ideal calibration we would expect a very sharp peak centered around $0 \mathrm{keV}$. The averaged value of $\Delta S$ over all pixels for the energies given in the previous paragraph is shown in Fig. 15. We can see that $\overline{\Delta S}$ 


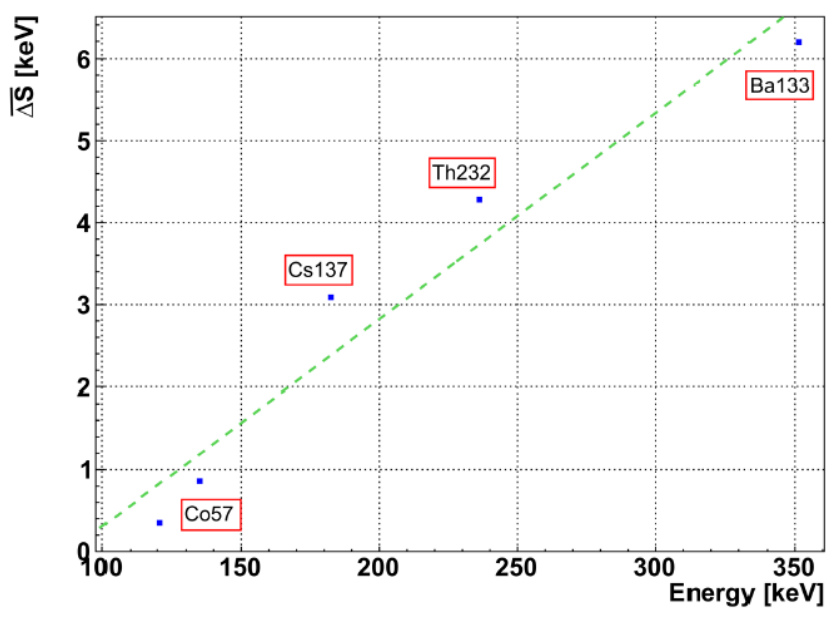

Fig. 15 Data points: averaged $\Delta S$ for peak energies above the pixel-by-pixel calibration range; the green line is a linear fit to the data. Statistical errors are very small and thus not visible

increases with energy (Fig. 15). We performed an additional calibration step to cope with this effect. It turned out that using a linear fit (green line) for the given data points and to subtract the value of the linear function from the regular TOT calibration curve leads to a significant improvement at energies beyond $120 \mathrm{keV}$. The positive effect on the peak position and the energy resolution are discussed in the next section.

\section{Tracking of electrons}

In order to investigate the performance of neural networks on experimental data, we performed the following experiment with a ${ }^{232} \mathrm{Th}$ source: About $35 \%$ of the photon flux radiated by natural ${ }^{232} \mathrm{Th}$ are $2.614 \mathrm{MeV}$ photons from ${ }^{208} \mathrm{Tl}$ which is the last nuclide in the natural decay chain of ${ }^{232} \mathrm{Th}$. In the CdTe sensor such photons can be Compton-scattered, which produces single electrons, or generate electron-positron pairs with a total kinetic energy of $1.588 \mathrm{MeV}$. Apparently, electron-positron pairs starting at one point produce a similar pattern to the two electrons in $0 \nu \beta \beta$ and hence electron-positron tracks can be used as a substitute to verify experimentally the pattern recognition power of the Timepix in a $0 \nu \beta \beta$ experiment.

In the actual experimental setup (Fig. 16) we used a ${ }^{232} \mathrm{Th}$ source (1) with an activity of $14.8 \mathrm{MBq}$ which was placed $40 \mathrm{~cm}$ away from the detector (2). The geometry was chosen in such a way that the photon momentum is approximately parallel to the sensor layer and every $0.3 \mathrm{~s}$ one or two pair production events are expected to occur. The detector was fixed onto a lead block and surrounded by a light shield box (3) to shield the detector from optical photons and by lead (4) for additional gamma shielding. We recorded and evaluated 34.4 hours of data. This gives us 3646025 events com-

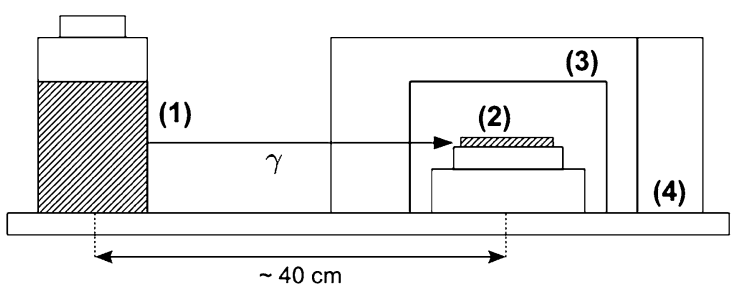

Fig. 16 A scheme of the experimental setup for the ${ }^{232}$ Th measurement. (1) The ${ }^{232} \mathrm{Th}$ source, (2) the sensor layer, (3) the cardboard and (4) the lead wall. The distance between the source and the detector was about $40 \mathrm{~cm}$

prising 606813 electron events (both single and pair production) after the rejection of $\alpha$-particle and muon events.

Such events are clusters on the pixel matrix as shown in Fig. 2 and Fig. 3. A cluster is a set of triggered pixels which are direct neighbors with each other. The energy deposited in a particular cluster is calculated as the sum over the energy depositions in all individual pixels belonging to the particular cluster. As electrons in the $\mathrm{MeV}$ energy range always trigger more than one pixel, all energy spectra in this section are spectra of such clusters, which means that every entry in the spectrum is due to a cluster and not to the energy deposition in a single pixel. We divided the data into two packages. We used the first to check our data analysis and the energy resolution. On the second package we tested the artificial neural networks.

\subsection{Energy resolution of electron-positron pairs at $1.6 \mathrm{MeV}$}

The energy spectrum of events in the region of interest (Fig. 17) consists of Compton background and the pair production peak at about $1.6 \mathrm{MeV}$. At this energy most of the event clusters consist of about 14-17 pixels in average. The energy resolution $\left(\frac{\sigma}{E}\right)$ at the peak is $2.2 \%$ (red) and the reconstructed peak energy is $1614.1 \mathrm{MeV}$ if the regular calibration curve is used (Eq. (5)). If we apply the additional calibration step explained in the previous section, the reconstructed peak position is $1589.1 \mathrm{MeV}$ (blue) and the energy resolution improves to $1.6 \%$ which is twice the value that we would expect from simulation.

We estimate that the reduced resolution is due to the weak energy calibration at high energies. The calibration data cover energies up to $130 \mathrm{keV}$ with high statistics and energies up to $350 \mathrm{keV}$ with low statistics. From the results presented in Fig. 15 we see that the extrapolation is not optimal. On the other hand we know from simulation that electron tracks can lead to energy deposition in a pixel of about $400 \mathrm{keV}$. Hence, a new method is needed to calibrate the detector up to these energies. One possibility to do so is to illuminate the detector with a tightly focused laser beam which is triggered to shot pulses with a precisely known amount of photons into one pixel during one frame. For this paper we 


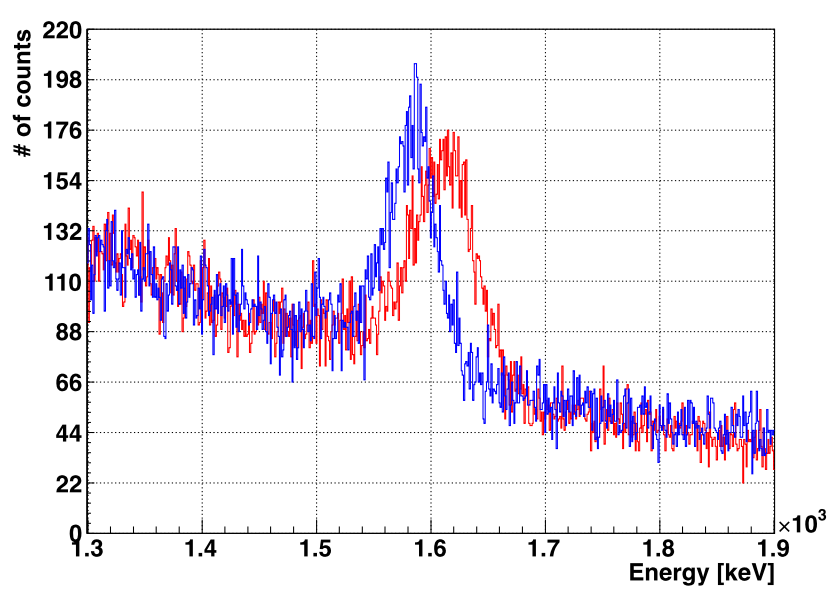

Fig. 17 Measured energy spectrum of pair production and Compton scattering events before (red) and after (blue) applying the additional high energy calibration

take the energy resolution as it is since it is sufficient for the track investigations which are the main issue in this paper.

\subsection{Separation power of neural networks on electron-positron pairs and single electrons}

As next we applied artificial neural networks to the data. We divided the region of interest from $1200 \mathrm{keV}$ to $2000 \mathrm{keV}$ into eight equal intervals of $200 \mathrm{keV}$ width. For every interval an individual network i was trained and an optimal cut parameter $c_{i}$ chosen. Every event of the spectrum was classified by the appropriate network $\mathrm{i}$ and assigned a rating $R$. If the rating of an event $R$ is smaller than the cut parameter $c_{i}$, it is classified as a single electron event and vice versa.

The rating $R$ is not a real physical quantity and does not describe some kind of probability. To perform the reconstruction correctly, the network performance for the chosen cut parameters $c_{i}$ has to be taken into account. In fact, if a particular value $c_{i}$ is chosen, the network classifies a particular percentage of the single electron events $\left(\pi_{1}\right)$ and a particular percentage of the two electron events $\left(\pi_{2}\right)$ correctly. These values are determined in the network training process. If we call the true number of single electron events in a particular energy bin $\eta_{1}$ and the true number of two electron events $\eta_{2}$, then the number of events classified as two electron events $\kappa_{2}$ can be calculated as

$$
\begin{aligned}
\kappa_{2} & =\pi_{2} \cdot \eta_{2}+\left(1-\pi_{1}\right) \cdot \eta_{1} \\
& =\pi_{2} \cdot \eta_{2}+\left(1-\pi_{1}\right) \cdot\left(N-\eta_{2}\right)
\end{aligned}
$$

$N$ is the total number of events in a particular energy bin $\left(\eta_{1}+\eta_{2}=\mathrm{N}\right)$. Actually, $\kappa_{2}$ is the quantity that we obtain from the data by assigning a rating $\mathrm{R}$ to every event. ${ }^{7}$ Now

\footnotetext{
${ }^{7}$ As the ANNs are sensitive on statistical fluctuations, we used the average value of the 5 neighbor bins (to the left and right) to smooth the spectrum for the ANNs.
}

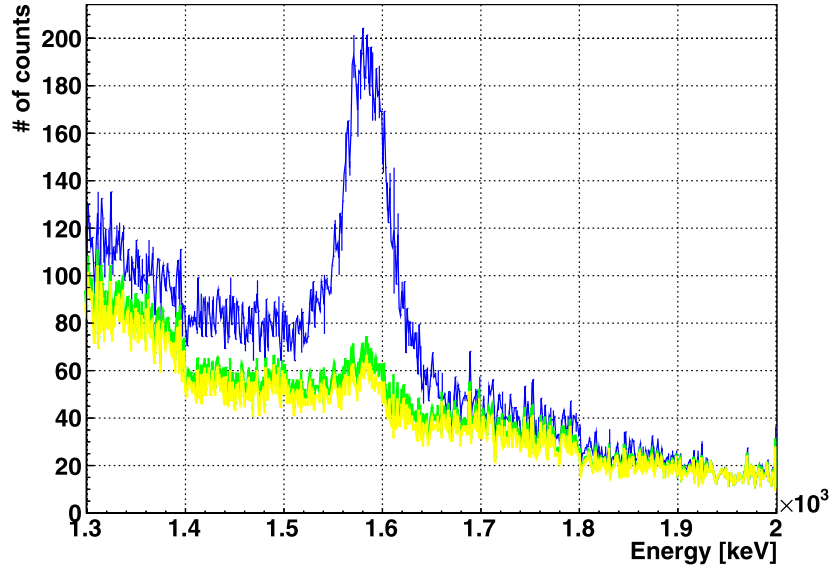

Fig. 18 Reconstructed electron spectrum in the region of interest; all events (blue), upper and lower limits (green/yellow) for events reconstructed as single electrons

the real number of single and two electron events can be calculated by inverting formula (9):

$\eta_{2}=\frac{1}{\pi_{1}+\pi_{2}-1}\left(\kappa_{2}-\left(1-\pi_{1}\right) \cdot N\right)$

In the reconstructed spectrum (Fig. 18) the area below the green/yellow lines belongs to the spectrum of Compton scattered photons and the area above the green/yellow lines (and below the blue line) to the pair-production events. The remaining events in the pair production spectrum below the peak energy are pair production events in which at least one of the particles escaped the sensor layer and therefore not the full energy was deposited in the sensor. The green line is the upper limit for the uncertainties in the event classification whereas the yellow line is the lower limit.

\section{Conclusions}

We investigated the applicability of the semiconductor pixel detector Timepix with a CdTe sensor for future use in the search for the $0 \nu \beta \beta$. A pixel-by-pixel energy calibration was performed in the range between $5 \mathrm{keV}$ and $120 \mathrm{keV}$ with high precision. For energies in the range $130 \mathrm{keV}$ to $350 \mathrm{keV}$ the calibration was performed with lower precision but still a resolution of $1.6 \%$ could be achieved at the pair production peak of ${ }^{208} \mathrm{Tl}$ at $1588.8 \mathrm{MeV}$. Nonetheless the results of the simulations indicate that the energy resolution can be improved by a factor of two.

We investigated the tracking capabilities of the Timepix detector in order to separate different types of events. The separation of muons and $\alpha$-particles from electrons is very pure. Further, a good separation of single electron tracks from electron-positron tracks could be reached by artificial neural networks. Thus, the tracking ability makes the 
Timepix detector an interesting candidate for future $0 v \beta \beta$ experiments.

One drawback may be the relatively large surface to volume ratio which almost forbids the choice of a fiducial volume with a certain distance from the surface. But it may turn out that due to the track ability fiducializing is not necessary. A second draw back is the enormous number of detectors that would be needed to achieve a reasonable detector mass. As long as other experimental approaches have not proven to be able to detect unambiguously the $0 v \beta \beta$ decay, the method proposed in this work might be kept as a possible solution.

Acknowledgements We would like to thank Steffen Taut, Daniel Gehre and Kai Zuber from the Technical University of Dresden for their support and giving us the opportunity to perform the experiments with ${ }^{232} \mathrm{Th}$ in the laboratory for radiochemistry.

This work was carried out within the Medipix collaboration and the COBRA collaboration. The authors thank both collaborations for their support.

Open Access This article is distributed under the terms of the Creative Commons Attribution License which permits any use, distribution, and reproduction in any medium, provided the original author(s) and the source are credited.

\section{References}

1. J. Schechter, J.W.F. Valle, Phys. Rev. D 25, 2951 (1982)

2. F. Simkovic, M.I. Krivoruchenko, A. Faessler, Prog. Part. Nucl. Phys. 66, 451-466 (2011)

3. H.V. Klapdor-Kleingrothaus, Nucl. Phys. B, Proc. Suppl. 145, 219-224 (2009)

4. S.R. Elliott, P. Vogl, Double beta decay. Annu. Rev. Nucl. Part. Sci. 52, 115-151 (2002)

5. J.J. Gomez-Cadenas et al., Sense and sensitivity of double beta decay experiments. J. Cosmol. Astropart. Phys. 2011, 06 (2011)

6. X. Llopart, R. Ballabriga, M. Campbell, L. Tlustos, W. Wong, Nucl. Instr. Methods A 581, 485-494 (2007)

7. K. Zuber, Phys. Let. B 519, 1-7 (2001)

8. S. Rahaman et al., Phys. Lett. B 703, 412-416 (2011)

9. M. Platkevic, J. Jakubek, Z. Vykydal et al., Nucl. Instr. and Meth. A 591, 245-247 (2008)

10. J. Giersch, A. Weidemann, G. Anton, Nucl. Instr. Methods A 509, $151-156$ (2003)

11. O.A. Ponkratenko, V.I. Tretyak, Yu.G. Zdesenko, Phys. At. Nucl. 63, $1282(2000)$

12. J. Jakubek, Nucl. Instr. Methods A 607, 192-195 (2008)

13. S. Nissen, Implementation of a Fast Artificial Neural Network Library (fann). Department of Computer Science (2003), University of Copenhagen (DIKU)

14. J. Jakubek et al., Nucl. Instr. Methods A 591, 155-158 (2008)

15. D. Greiffenberg, A. Fauler, A. Zwerger et al., J. Instrum. 6, C01058 (2011)

16. D. Turecek, J. Jakubek, Z. Vykydal et al., J. Instrum. 6, C01046 (2011) 